\title{
Pacific
}

Journal of

Mathematics

\section{CONCORDANCES OF METRICS OF POSITIVE SCALAR}

\section{CURVATURE}

\author{
PAWEL GAJER
}




\title{
CONCORDANCES OF METRICS OF POSITIVE SCALAR CURVATURE
}

\author{
Pawel Gajer
}

\begin{abstract}
Spaces of metrics of positive scalar curvature are studied modulo a concordance relation. It is shown that the set of concordance classes of metrics with positive scalar curvature on a closed manifold of dimension $\geq 6$ depends only on the dimension, the first StiefelWhitney class of the manifold, and the cokernel of a homomorphism $\pi_{2}\left(M^{n}\right) \rightarrow \widetilde{K O}\left(S^{2}\right)$. In addition, for every nonnegative integer $i$ the $i$ th concordance group of metrics of positive scalar curvature is defined and it is shown that for a spin manifold the group is nontrivial when $n+i=4 k+3,8 k, 8 k+1, k \geq 1$.
\end{abstract}

Two metrics $g_{0}$ and $g_{1}$ with positive scalar curvature on $M^{n}$ are concordant, written $g_{0} \cong g_{1}$, if there is a metric $g$ of positive scalar curvature on $M^{n} \times[0,1]$ such that $\left.g\right|_{M^{n} \times\{i\}}=g_{i}$ for $i=0,1$, and $g$ is a product near $M^{n} \times \partial[0,1]$. It is easy to see that concordance is an equivalence relation. The set of its equivalence classes on the space $\mathbb{P S C}\left(M^{n}\right)$ of metrics of positive scalar curvature on $M^{n}$ will be denoted by $\pi_{0}^{c}\left(\mathbb{P S C}\left(M^{n}\right)\right)$. For $n \geq 3$ the connected sum operation induces a group structure on $\pi_{0}^{c}\left(\mathbb{P S C}\left(S^{n}\right)\right)$ [G]. It will be proved that for every simply-connected spin manifold $M^{n}$ of dimension $n \geq 6$ the group acts freely and transitively on $\pi_{0}^{c}\left(\mathbb{P S C}\left(M^{n}\right)\right)$. This is a special case of the following result.

THEOREM 2.1. Let $M^{n}$ be a closed manifold of dimension $n \geq 6$. There exists a group depending only on the dimension, the first StiefelWhitney class of $M^{n}$, and the cokernel of the homomorphism

$$
\pi_{2}\left(M^{n}\right) \rightarrow \widetilde{K O}\left(S^{2}\right) \text { given by }[\varphi] \mapsto\left[\varphi^{*} T M^{n}\right]
$$

that acts freely and transitively on the set $\pi_{0}^{c}\left(\mathbb{P S C}\left(M^{n}\right)\right)$.

The group occurring in the statement of Theorem 2.1 is essentially Hajduk's obstruction group for the existence of metrics of positive scalar curvature (for more details see $\S 2$ and [H2]).

Every smooth map $g:[0,1] \rightarrow \mathbb{P S C}\left(M^{n}\right)$ induces a concordance between $g(0)$ and $g(1)$ [GL2, Proposition 4.43]. In other words, 
there is a surjective map $\pi_{0}\left(\mathbb{P S C}\left(M^{n}\right)\right) \rightarrow \pi_{0}^{c}\left(\mathbb{P S C}\left(M^{n}\right)\right)$. For every $i \geq 1$, there exists an $i$ th-dimensional counterpart $\pi_{i}\left(\mathbb{P S C}\left(M^{n}\right), g\right) \rightarrow$ $\pi_{i}^{c}\left(\mathbb{P S C}\left(M^{n}\right), g\right)$ of the map, which is a group homomorphism. The groups $\pi_{i}^{c}\left(\mathbb{P S C}\left(M^{n}\right), g\right)$ are called concordance groups of positive scalar curvature metrics on $M^{n}$, with the base metric $g$. The following theorem is one of the main results of this paper.

THEOREM 3.1. If $M^{n}$ is a closed spin manifold, then $\pi_{i}^{c}\left(\mathbb{P S C}\left(M^{n}\right), g\right)$ $\neq 0$ for every $n+i=4 k+3,8 k, 8 k+1, k \geq 1$.

This is a refinement of the following theorem due to Hitchin: if $M^{n}$ is a closed spin manifold, then $\pi_{i}\left(\mathbb{P S C}\left(M^{n}\right)\right) \neq 0$ for $i=0,1$ and $n+i=8 k, 8 k+1, k \geq 1$.

The paper is organized as follows. In $\S 1$ the notion of a handle metric is introduced and it is proved that an arbitrary metric of positive scalar curvature is isotopic to one that decomposes into a sum of a handle metric and a concordance (see Theorem 1.1). Section 2 contains the proof of Theorem 2.1. Concordance groups of positive scalar curvature metrics are studied in $\S 3$.

I would like to thank Marek Lewkowicz for many valuable conversations on deformations of metrics of positive scalar curvature. A special thanks is due to Bogusław Hajduk for sharing with me his ideas on obstruction groups for the existence of metrics with positive scalar curvature and for encouraging me during the preparation of this paper.

1. Deformations of $\mathbb{P S C}$ metrics. In the section the notions of handle and GL metrics are introduced and the following results on deformations of positive scalar curvature metrics are proved.

THEOREM 1.1. Let $W^{n+1}$ be a bordism between closed manifolds $M^{n}$ and $N^{n}$. If $W^{n+1}$ has a handle decomposition without handles of codimension less than three, then every metric $g$ on $W^{n+1}$ of positive scalar curvature and a product near the boundary is isotopic to one which decomposes into the sum $g_{H} \cup c$ of a handle metric $g_{H}$ and a concordance $c$ between $\left.g_{H}\right|_{N^{n}}$ and $\left.g\right|_{N^{n}}$.

THEOREM 1.2. Let $V^{i}$ be a submanifold of $M^{n} \backslash \partial M^{n}$ of codimension greater than two with a trivial normal bundle. Every metric of positive scalar curvature on $M^{n}$ is isotopic to one which is GL along $V^{i}$. 
In this paragraph GL metrics along submanifolds are defined. Handle metrics will appear later as a by-product of the following construction of GL metrics. Let $V^{i}$ and $M^{n}$ be as in Theorem 1.2. Let us first consider the situation where $V^{i}$ is a point $x$. If $n \geq 3$, then it is possible to deform an arbitrary metric of positive scalar curvature on $M^{n}$ in a neighborhood $D$ of $x$ by the following Gromov-Lawson construction (cf. [GL1, the proof of Theorem A]). First we take a hypersurface of revolution $L$ in $D \times \mathbb{R}_{+}$with a metric of positive scalar curvature on it which at the right end of $L$ is of the form $g_{\text {can }}^{n-1}+d t^{2}$, where $g_{\text {can }}^{k}$ is the standard metric on the unit sphere $S^{k}$. Then we glue $\mathbb{D}^{n}$ to the end of $L$ and extend the metric from $L$ to $\mathbb{D}^{n}$ by the "torpedo" metric $g_{t p}^{n}$. The metric obtained in this way on $M^{n}$ will be called $G L$ around $x$. Assume now that $V^{i}$ is a closed submanifold of $M^{n}$. We say that a metric of a positive scalar curvature on $M^{n}$ is $G L$ along $V^{i}$ if there is a neighborhood of $V^{i}$ in $M^{n}$ such that the metric induced on the neighborhood is of the form $g_{V}+g_{\mathrm{GL}}^{n-i}$, where $g_{\mathrm{GL}}^{n-i}$ is a GL metric on the disk $\mathbb{D}^{n-i}$ and $g_{V}$ is a metric on $V^{i}$ (not necessarily of positive scalar curvature). If $V^{i}$ has a nonempty boundary $\partial V^{i}$, then the GL metric along $V^{i}$ is defined in the following way. Take $\varepsilon>0$ such that the exponential map on $\varepsilon$-disk bundles $\nu_{\varepsilon}\left(\partial V^{i}\right)$ and $\nu_{\varepsilon}\left(V^{i}\right)$ of the normal bundles of $\partial V^{i}$ and $V^{i}$ in $M^{n}$ is an embedding. In the sequel these disk bundles will be identified with their images by the exponential map. The normal bundle to $\partial V^{i}$ splits into the sum $\left.\nu\left(V^{i}\right)\right|_{\partial V^{i}} \oplus \operatorname{span}(X)$, where $X$ is a field of outward vectors on $\partial V^{i}$ orthogonal to $\nu\left(V^{i}\right)$ and $\operatorname{span}(X)$ is the line bundle on $\partial V^{i}$ induced by $X$. Consider a neighborhood $\nu_{+, \varepsilon}\left(\partial V^{i}\right) \cup \nu_{\varepsilon}\left(V^{i}\right)$ of $V^{i}$ in $M^{n}$, with $\nu_{+, \varepsilon}\left(\partial V^{i}\right)=\left\{(v, t X) \in\left(\nu\left(V^{i}\right) \oplus \operatorname{span}(X)\right) \cap \nu_{\varepsilon}\left(\partial V^{i}\right): t>0\right\}$. A metric $g$ of positive scalar curvature on $M^{n}$ is $G L$ along $V^{i}$ if there exists $\varepsilon_{0} \in(0, \varepsilon)$ such that the restriction of $g$ to the neighborhood $\nu_{+, \varepsilon_{0}}\left(\partial V^{i}\right) \cup \nu_{\varepsilon_{0}}\left(V^{i}\right)$ of $V^{i}$ is of the form

$$
\left(\left.g\right|_{\partial V^{i}}+\left.g_{\mathrm{GL}}^{n-i+1}\right|_{D_{+}^{n-i+1}}\right) \cup\left(g_{V}+g_{\mathrm{GL}}^{n-i}\right) .
$$

The proof of Theorem 1.2 in the case when $V^{i}$ is a closed submanifold of $M^{n}$ follows exactly the proof of Theorem $2^{\prime}$ in [G]. When $V^{i}$ has a nonempty boundary one has to deform a given metric on $\nu_{+, \varepsilon}\left(\partial V^{i}\right)$ and $\nu_{\varepsilon}\left(V^{i}\right)$ at the same time using the technique of the proof of Theorem $2^{\prime}$.

Proof of Theorem 1.1. Let $W^{n+1}$ be as in Theorem 1.1. The modification of a given metric of positive scalar curvature on $W^{n+1}$ to a 
handle one (which will be defined along the proof) will have an inductive character; with induction on the number of handles of $W^{n+1}$. The inductive step follows.

Let $W^{n+1}=\left(M^{n} \times I\right) \cup_{j} H^{i+1}$, where $j: \partial\left(\mathbb{D}^{i+1}\right) \times \mathbb{D}^{n-i} \rightarrow M^{n} \times$ $\{1\}$ is the gluing map for $H^{i+1}$, and let $g$ be a metric of positive scalar curvature on $W^{n+1}$ product in a neighborhood of the boundary. Choose $\gamma>0$ such that $g$ is product in the collar $M^{n} \times[0, \gamma]$ of $M^{n} \times\{0\}$ in $M^{n} \times I$. By an abuse of notation $\mathbb{D}^{i+1}$ will denote the disk $\left(\mathbb{D}^{i+1} \times\{0\}\right) \cup_{j}\left(j\left(\partial\left(\mathbb{D}^{i+1} \times\{0\}\right) \times(\gamma / 2,1]\right)\right)$, where $\mathbb{D}^{i+1} \times\{0\}$ is the left disk of $H^{i+1}$. We can apply Theorem 1.2 to the disk $\mathbb{D}^{i+1}$ and deform $g$ in a neighborhood $\nu_{+, \varepsilon}\left(\partial \mathbb{D}^{i+1}\right) \cup\left(\mathbb{D}^{i+1}\right)$ of $\mathbb{D}^{i+1}$ to a metric of the form

$$
\left(\left.g\right|_{\partial D^{i+1}}+\left.g_{\mathrm{GL}}^{n-i+1}\right|_{D_{+}^{n-i+1}}\right) \cup\left(\left.g\right|_{D^{i+1}}+g_{\mathrm{GL}}^{n-i}\right) .
$$

There exist: $\mu>0$ and $\varepsilon_{0} \in(0, \varepsilon)$ such that $g$ restricted to $\nu_{+, \varepsilon_{0}}\left(\partial \mathbb{D}^{i+1}\right) \cup \nu_{\varepsilon_{0}}\left(\mathbb{D}^{i+1}\right)$ is of the form

$$
\left(\left.g\right|_{\partial D^{i+1}}+\left.\mu g_{\text {can }}^{n-i+1}\right|_{D_{+}^{n-i+1}}\right) \cup\left(\left.g\right|_{D^{i+1}}+\mu g_{t p}^{n-i}\right) .
$$

If $\mu$ is small enough, then the metric can be isotoped to

$$
g^{\prime}=\left(g_{\mathrm{can}}^{i}+\left.\mu g_{\mathrm{can}}^{n-i+1}\right|_{D_{+}^{n-i+1}}\right) \cup\left(g_{t p}^{i+1}+\mu g_{t p}^{n-i}\right) .
$$

This metric can be described in another way as follows. Embed $\mathbb{D}^{i+1}$ in $\mathbb{R}^{i+2}$ such that the induced on $\mathbb{D}^{i+1}$ metric will be "torpedo". Consider the embedding induced by the sequence of inclusions

$$
\begin{aligned}
\mathbb{D}^{i+1} \times(-\infty, 0] & \subset \mathbb{R}^{i+2} \times(-\infty, 0] \\
& \subset \mathbb{R}^{i+2} \times \mathbb{R} \subset \mathbb{R}^{i+2} \times \mathbb{R} \times \mathbb{R}^{n-i+1}=\mathbb{R}^{n+4}
\end{aligned}
$$

and put $P=\left\{x \in \mathbb{R}^{n+4} \mid \operatorname{dist}\left(x, \mathbb{D}^{i+1} \times(-\infty, 0]\right)=\mu\right\}$. There exists $\delta>0$ such that the metric induced by the Euclidean metric of $\mathbb{R}^{n+4}$ on $\widehat{P}=P \cap\left(\mathbb{R}^{i+2} \times(-\delta, 0] \times \mathbb{R}^{n-i+1}\right)$ will coincide with $g^{\prime}$ if we smooth the corner along $P \cap\left(\mathbb{R}^{i+2} \times\{0\} \times \mathbb{R}^{n-i+1}\right)$. Let us now embed $\mathbb{R}^{i+2} \times \mathbb{R} \times \mathbb{R}^{n-i+1}$ into $\mathbb{R}^{i+2} \times \mathbb{R} \times \mathbb{R}^{i+1} \times \mathbb{R}$ and rotate $\mathbb{D}^{i+1}$ in this space around $\mathbb{R}^{i+2} \times\{0\} \times \mathbb{R}^{n-i+1} \times\{0\}$ by $\pi / 2$ radians. The trace $S$ of this rotation has as a boundary $D_{L} \cup D_{R}$, where $D_{L}$ and $D_{R}$ are the images of $\mathbb{D}^{i+1}$ in $\mathbb{R}^{n+4}$ before and after rotation. Let $T$ be a manifold obtained from $\mathbb{D}^{i+1} \times(-\infty, 0]$ by gluing to $\mathbb{D}^{i+1} \times\{0\}$ the trace of the rotation $S$ along $D_{L}$, and then gluing to the resulting manifold the product $\left(\left(\partial \mathbb{D}^{i+1} \times(-\infty, 0]\right) \cup \mathbb{D}^{i+1}\right) \times[0, \delta)$ along $\left(\partial \mathbb{D}^{i+1} \times(-\infty, 0]\right) \cup D_{R}$, and smoothing the resulting corners. Consider $P^{\prime}=\left\{x \in \mathbb{R}^{n+4} \mid \operatorname{dist}(x, T)=\mu\right\}$. The rotation of $\mathbb{D}^{i+1}$ induces 
an isotopy between $g^{\prime}$ and the metric induced by the Euclidean metric of $\mathbb{R}^{n+4}$ on $P^{\prime}$. Let us extend the isotopy to an isotopy between the GL metric along $\mathbb{D}^{i+1}$ and a metric $\hat{g}$. There exists $\varepsilon_{1} \in(0, \delta)$ such that the metric $g_{H}$ induced on $\nu_{+, \varepsilon_{0}}\left(\partial \mathbb{D}^{i+1}\right) \cup \nu_{\varepsilon_{0}}\left(\partial \mathbb{D}^{i+1} \times\left[0, \varepsilon_{1}\right)\right)$ by $\hat{g}$ has positive scalar curvature and is product near the boundary. The metric induced by $\hat{g}$ on the complement of $\nu_{+, \varepsilon_{0}}\left(\partial \mathbb{D}^{i+1}\right) \cup$ $\nu_{\varepsilon_{0}}\left(\partial \mathbb{D}^{i+1} \times\left[0, \varepsilon_{1}\right)\right)$ is a concordance $c$ between $\left.g_{H}\right|_{N^{n}}$ and $\left.g\right|_{N^{n}}$, where $N^{n}=\partial\left(W^{n+1}\right) \backslash M^{n}$. Hence $g$ is isotopic to a metric $g_{H} \cup c$. The metric $g_{H}$ is determined by $g_{M}=\left.g\right|_{M^{n} \times\{0\}}$ and $H^{i+1}$ in the unique way only up to isotopy. Hence every metric isotopic to $g_{H}$ will be called in the sequel the handle metric induced by $g_{M}$ and the handle $H^{i+1}$. More generally, if $\left(M^{n} \times I\right) \cup H^{\lambda_{0}} \cup \cdots \cup H^{\lambda_{k}}$ is a handle decomposition on $M^{n}$ of a bordism $W^{n+1}$ without handles of codimension less than three, then the inductive usage of the handle metric construction produces a metric of positive scalar curvature on $W^{n+1}$ which we shall call the handle metric induced by $g$ and the handle decomposition $\left(M^{n} \times I\right) \cup H^{\lambda_{0}} \cup \cdots \cup H^{\lambda_{k}}$.

The next theorem is a straightforward consequence of the handle metric construction.

SuRgery TheOREM ([GL1, SY, G]). Let $\left(M^{n}, g\right)$ be a closed Riemannian manifold with positive scalar curvature and let $W^{n+1}$ be a bordism between $M^{n}$ and $N^{n}$ such that $W^{n+1}$ admits a handle decomposition on $M^{n}$ without handles of codimension less than three, then there exists a metric with positive scalar curvature on $W^{n+1}$, which extends $g$ and is product on a collar of $M^{n} \cup N^{n}$.

The following result is a version of Theorem 1.1 for manifolds with boundary.

THEOREM 1.3. Let $M^{n}$ be a closed manifold with a handle decomposition with all handles of codimension greater than two. Then every concordance $g$ between metrics $g_{0}, g_{1}$ of positive scalar curvature on $M^{n}$ is isotopic to a metric of the form $\left.\left(g_{H}+d t^{2}\right) \cup c \cup g\right|_{M_{\leq 2}}$, where $M_{\leq 2}$ is the sum of handles of codimension $\leq 2$ and $c$ is a concordance on $\partial M_{\leq 2}$.

The proof of Theorem 1.3 goes along the lines of the proof of Theorem 1.1. Let $M^{n}$ and $g$ be as in Theorem 1.3. It is possible to extend $g$ from $M^{n} \times I$ to a metric $\hat{g}$ of positive scalar curvature on 
$M^{n} \times \mathbb{R}$ putting on $M^{n} \times(-\infty, 0]$ and $M^{n} \times[1,+\infty)$ the appropriate product metrics. Then, using Theorem 1.2 , one can inductively deform $\hat{g}$ along the products $\mathbb{D}^{\lambda_{i}} \times \mathbb{R}$, where $\mathbb{D}^{\lambda_{i}}$ is an extended left disk of $h^{\lambda_{i}}$, getting the required isotopy.

2. Concordances of $\mathbb{P S C}$ metrics. One of the basic corollaries of the Surgery Theorem is that existence of a positive scalar curvature metric on a manifold $M^{n}$ depends on the 2-connected bordism class of $M^{n}$. In this section it is shown that the set of concordance classes of metrics with positive scalar curvature on $M^{n}$ depends only on the dimension, the first Stiefel-Whitney class of $M^{n}$, and the cokernel of a homomorphism $\pi_{2}\left(M^{n}\right) \rightarrow \widetilde{K O}\left(S^{2}\right)$.

THEOREM 2.1. Let $M^{n}$ be a closed manifold of dimension greater than five. There exists a group depending only on the dimension, the first Stiefel-Whitney class of $M^{n}$, and the cokernel of the homomorphism

$$
\pi_{2}\left(M^{n}\right) \rightarrow \widetilde{K O}\left(S^{2}\right) \text { given by }[\phi] \mapsto\left[\phi^{*} T M^{n}\right]
$$

that acts on the set $\pi_{0}^{c}\left(\mathbb{P S C}\left(M^{n}\right)\right)$ in a transitive and free way.

When $M^{n}$ is simply-connected and spin, the group is $\pi_{0}^{c}\left(\mathbb{P S C}\left(S^{n}\right)\right)$. In general the group is essentially Hajduk's obstruction group for the existence of metrics of positive scalar curvature, which construction will be described later.

A bordism $W^{n+1}$ between closed manifolds $M^{n}$ and $N^{n}$ is called 2-connected if the groups $\pi_{i}\left(W^{n+1}, M^{n}\right)$ and $\pi_{i}\left(W^{n+1}, N^{n}\right)$ are trivial for $i=1,2$.

Proposition 2.2. If $M^{n}$ and $N^{n}$ are 2-connected bordant closed manifolds of dimension greater than four, then the sets of concordance classes of metrics of positive scalar curvature on $M^{n}$ and $N^{n}$ are in a bijective correspondence.

The proof of Proposition 2.2 is based on the following lemma.

LEMMA 2.3. Let $W^{n+1}$ be a bordism between $M^{n}$ and $N^{n}$ with a handle decomposition on $M^{n}$ without handles of codimension less than three, and let $g_{0}$ and $g_{1}$ be two metrics of positive scalar curvature on $W^{n+1}$ product in a collar of the boundary. If $\left.g_{0}\right|_{M^{n}}$ and $\left.g_{1}\right|_{M^{n}}$ are concordant, then the metrics $\left.g_{0}\right|_{N^{n}}$ and $\left.g_{1}\right|_{N^{n}}$ are concordant as well.

Proof. Let $c$ be a concordance between the metrics $\left.g_{0}\right|_{M^{n}}$ and $\left.g_{1}\right|_{M^{n}}$. The metric $g_{0} \cup c \cup g_{1}$ on $W^{n+1} \cup_{M^{n}}\left(M^{n} \times I\right) \cup M^{n}\left(-W^{n+1}\right)$ has 
positive scalar curvature and is a product near the boundary. Since $W^{n+1}$ is the 2-connected bordism, the manifold

$$
W^{n+1} \cup_{M^{n}}\left(M^{n} \times I\right) \cup_{M^{n}}\left(-W^{n+1}\right)
$$

is relatively 2-connected bordant to $N^{n} \times I$. By the Surgery Theorem, the above bordism and the metric $g_{0} \cup c \cup g_{1}$ induce a concordance between $\left.g_{0}\right|_{N^{n}}$ and $\left.g_{1}\right|_{N^{n}}$. This completes the proof of Lemma 2.3.

Proof of Proposition 2.2. Let $W^{n+1}$ be a 2-connected bordism between $M^{n}$ and $N^{n}$. By Morse-Smale theory, there are handle decompositions of $W^{n+1}$ on $M^{n}$ and on $N^{n}$ without handles of codimension less than three. Thus, every metric $g$ of positive scalar curvature on $M^{n}$ induces a metric $S(g)$ on $N^{n}$, and conversely, every metric $g^{\prime}$ on $N^{n}$ induces a metric $S^{-1}\left(g^{\prime}\right)$ on $M^{n}$. By Lemma $2.3, S$ and $S^{-1}$ depend only on concordance classes of metrics of positive scalar curvature and are inverse to one another.

For every smooth manifold $M^{n}$ and a positive integer $i<\operatorname{dim} M^{n}$ the kernel of the homomorphism

$$
\pi_{i}\left(M^{n}\right) \rightarrow \widetilde{K O}\left(S^{i}\right), \quad[\varphi] \mapsto\left[\varphi^{*} T M^{n}\right]
$$

describes the part of $\pi_{i}\left(M^{n}\right)$ which can be killed by surgery. For $i=1$ it is the first Stiefel-Whitney class of $M^{n}$. For $i=2$ it is the second Stiefel-Whitney class of $M^{n}$, if $M^{n}$ is a simply-connected manifold.

Let $\pi$ be a finitely presentable group and let $\omega=\left(\omega_{1}, \omega_{2}\right)$ where $\omega_{1} \in \operatorname{Hom}(\pi, \mathbb{Z} / 2 \mathbb{Z})$ and $\omega_{2} \in \mathbb{Z} / 2 \mathbb{Z}$. We say that $M^{n}$ is a $(\pi, \omega)$ manifold if $\pi_{1}\left(M^{n}\right) \cong \pi, \omega_{1}\left(M^{n}\right)=\omega_{1}$, and the homomorphism $\pi_{2}\left(M^{n}\right) \rightarrow \widetilde{K O}\left(S^{2}\right)$ is an isomorphism when $\pi_{2}=1$, and $\pi_{2}\left(M^{n}\right)$ is trivial when $\omega_{2}=0$. If $M^{n}$ is a closed manifold of dimension $\geq 5$, then surgery killing the kernel of $\pi_{2}\left(M^{n}\right) \rightarrow \widetilde{K O}\left(S^{2}\right)$ induces a 2-connected bordism between $M^{n}$ and a $(\pi, \omega)$-manifold with $\pi=$ $\pi_{1}\left(M^{n}\right), \omega_{1}=\omega_{1}\left(M^{n}\right)$, and $\omega_{2}$ depending on the cokernel of the homomorphism $\pi_{2}\left(M^{n}\right) \rightarrow \widetilde{K O}\left(S^{2}\right)$.

The remaining part of the section is devoted to a proof of the following result.

THEOREM 2.4. For every $(\pi, \omega)$-manifold $M^{n}$ of dimension $n \geq$ 6 there is a transitive and free action of a group $\pi_{0}^{c}(n, \pi, \omega)$ on $\pi_{0}^{c}\left(\mathbb{P S C}\left(M^{n}\right)\right)$.

It is clear that Proposition 2.2 together with Theorem 2.4 imply Theorem 2.1. 
$\pi_{0}^{c}(n, \pi, \omega)$ are Hajduk's obstruction groups for the existence of metrics of positive scalar curvature on $(\pi, \omega)$-manifolds. Originally Hajduk defined them for spin manifolds. The following few paragraphs describe adaptation of his construction to $(\pi, \omega)$-manifolds.

For every presentation $\alpha$ of $\pi$, a homomorphism $\pi \rightarrow \mathbb{Z} / 2 \mathbb{Z}$, an element of $\mathbb{Z} / 2 \mathbb{Z}$, and a number $n \geq 5$ there will be defined a $(\pi, \omega)$ manifold with boundary $\mathbb{D}^{n}(\pi, \alpha, \omega)$ such that $\mathbb{D}^{n}(\pi, \alpha, \omega)=\mathbb{D}^{n}$ when $\pi$ and $\omega$ are trivial and the following result holds.

LEMMA 2.5 ([H2]). Let $M^{n}$ be a $(\pi, \omega)$-manifold of dimension $\geq 5$. Then for an arbitrary presentation $\alpha$ of $\pi$ there is an embedding $\varphi: \mathbb{D}^{n}(\pi, \alpha, \omega) \rightarrow M^{n}$ and a handle decomposition of $M^{n}$ such that $\varphi$ maps the canonical handle decomposition of $\mathbb{D}^{n}(\pi, \alpha, \omega)$ onto the union of all handles of codimension less than three.

Later on we will see that if $\mathbb{T}^{n}(\pi, \alpha, \omega)$ is the doubling of $\mathbb{D}^{n}(\pi, \alpha, \omega)$, then the set of concordance classes of metrics of positive scalar curvature on $\mathbb{T}^{n}(\pi, \alpha, \omega)$ is a group, and it does not depend on the presentation $\alpha$ of $\pi$.

This paragraph contains the definition of $\mathbb{D}^{n}(\pi, \alpha, \omega)$. Assume that $n \geq 5$ and let $\pi$ be a group with a presentation $\alpha=\left(a_{1}, a_{2}, \ldots\right.$, $\left.a_{t} \mid r_{1}, r_{2}, \ldots, r_{s}\right)$ and $\omega_{1} \in \operatorname{Hom}(\pi, \mathbb{Z} / 2 \mathbb{Z})$. We can rearrange the order of the generators such that

$$
\omega_{1}\left(a_{i}\right)= \begin{cases}0 & \text { for } 1 \leq i \leq l \\ 1 & \text { for } i+1 \leq i \leq t\end{cases}
$$

It is well known that for every $n \geq 1$ there are only two, up to isomorphism, $n$-dimensional vector bundles $\varepsilon^{n}, \gamma^{n}$ over $S^{1}$. Let $\mathbb{D}\left(\varepsilon^{n}\right)$ and $\mathbb{D}\left(\gamma^{n}\right)$ be the unit disk bundles associated with $\varepsilon^{n}$ and $\gamma^{n}$ respectively. The boundary connected sum of $l$ copies of $\mathbb{D}\left(\varepsilon^{n-1}\right)$ and $t-l$ copies of $\mathbb{D}\left(\gamma^{n-1}\right)$ is a manifold $V_{1}$ with the fundamental group free of $l$ generators. For every relator $r_{i}$ of $\pi$ there is a smooth embedding $\tilde{r}_{i}: S^{1} \rightarrow \partial V_{1}$, whose image in $\pi_{1}\left(V_{1}\right)$ coincides with $r_{i}$. Since $\omega_{1}$ or $r_{i}$ is trivial, the normal bundle to $r_{i}\left(S^{i}\right)$ in $\partial V_{1}$ is trivial. Let $R_{i}$ be a trivialization of the normal bundle. The manifold $V_{2}$ is obtained from $V_{1}$ by attaching the trivial disk bundle over $\mathbb{D}^{2}$ to $\partial V_{1}$ along $R_{i}$ maps. The diffeomorphism type of $V_{2}$ does not depend on the choice of the trivializations $R_{i}$. For $\omega_{2}=0$ let $\mathbb{D}^{n}(\pi, \alpha, \omega)=V_{2}$ and for $\omega_{2}=1$ let $\mathbb{D}^{n}(\pi, \alpha, \omega)$ be the boundary connected sum of $V_{2}$ with a nontrivial $(n-2)$-dimensional disk bundle over $S^{2}$ (for 
$n \geq 3$ there are only two isomorphism classes of vector bundles over $\left.S^{2}\right)$.

Let $\mathbb{T}^{n}(\pi, \alpha, \omega)=\mathbb{D}^{n}(\pi, \alpha, \omega) \cup_{\partial}\left(-\mathbb{D}^{n}(\pi, \alpha, \omega)\right)$ be the doubling of $\mathbb{D}^{n}(\pi, \alpha, \omega)$.

LEMMA 2.6 ([H2]). If $n \geq 6$ and $\alpha_{0}, \alpha_{1}$ are presentations of $a$ group $\pi$, then the sets of concordance classes of metrics of positive scalar curvature on $\mathbb{T}^{n}\left(\pi, \alpha_{0}, \omega\right)$ and $\mathbb{T}^{n}\left(\pi, \alpha_{1}, \omega\right)$ are in a bijective correspondence.

Lemma 2.6 follows from Proposition 2.2 and the fact that for $n \geq 6$ and two arbitrary presentations $\alpha_{1}, \alpha_{2}$ of $\pi$ the spaces $\mathbb{T}^{n}\left(\pi, \alpha_{1}, \omega\right)$ and $\mathbb{T}^{n}\left(\pi, \alpha_{2}, \omega\right)$ are 2 -connected bordant one to another (for details see $[\mathbf{H 2}])$. In the sequel we use the notation $\pi_{0}^{c}(n, \pi, \omega)$ for the set of metrics of positive scalar curvature on $\mathbb{T}^{n}(\pi, \alpha, \omega)$.

The group structure on $\pi_{0}^{c}(n, \pi, \omega)$ is induced by the following operation. Let $s_{0}, s_{1}$ be metrics of positive scalar curvature on $\mathbb{T}^{n}(\pi, \alpha, \omega)$. The handle metric $g_{\text {can }}$ induced by the canonical handle decomposition of $\mathbb{D}^{n}(\pi, \alpha, \omega)$ will be called canonical. By Theorem $1.1, s_{0}$ and $s_{1}$ are isotopic to metrics of the form $g_{\text {can }} \cup c_{0}^{\prime} \cup g_{\text {can }}$ and $g_{\text {can }} \cup c_{1} \cup g_{\text {can }}$ respectively. Define $\left[s_{0}\right] \circ\left[s_{1}\right]=\left[g_{\text {can }} \cup \mathcal{c}_{0} \cup c_{1} \cup g_{\text {can }}\right]$. The operation is well defined by Theorem 1.3. The neutral element is given by the trivial concordance $\left.g_{\text {can }}\right|_{\partial D_{n}}(\pi, \alpha, \omega)+d t^{2}$ on $\partial D_{n}(\pi, \alpha, \omega)$ itself. The inverse to $[g]$ is $[-g]$ by the following result.

Cancellation Lemma. Let $c$ be a concordance between metrics $g_{0}$ and $g_{1}$ of positive scalar curvature on a closed manifold $M^{n}$ of dimension greater than four and let $-c=\varphi^{*}(c)$ where

$$
\varphi:\left(M^{n} \times[-1,1]\right) \rightarrow\left(M^{n} \times[-1,1]\right):(x, t) \mapsto(x,-t) .
$$

Then the concordance $c \cup_{g_{1}}(-c)$ is relatively concordant to the trivial one $g_{0}+d t^{2}$.

Proof. Consider the product metric $g+d t^{2}$ on $\left(M^{n} \times I\right) \times I$ where $I=[-1,1]$. Smoothing the metric (cf. [G]) around the corners $M^{n} \times\{-1\} \times\{-1\}$ and $M^{n} \times\{-1\} \times\{1\}$ of $\left(M^{n} \times I\right) \times I$ produces the metric $c \cup_{g_{1}}(-c)$ on $M^{n} \times I=\partial\left(\left(M^{n} \times I\right) \times I\right) \backslash\left(M^{n} \times\{1\} \times\right.$ $(-1,1))$ and the product metric $g_{0}+d t^{2}$ on $M^{n} \times\{1\} \times I$. Take $\left(M^{n} \times[1,2]\right) \times[-0.5,0.5]$ with the product metric $g_{0}+d l^{2}+d t^{2}$ and glue it to $\left(M^{n} \times I\right) \times I$ identifying the common parts and smoothing the corners. Obtain in this way the metric, which gives the required relative concordance. 
By Theorem 1.1, an arbitrary metric $g$ of positive scalar curvature on a $(\pi, \omega)$-manifold $M^{n}$ is isotopic to a metric of the form $g_{H} U$ $c_{g} \cup g_{\text {can }}$ where $g_{H}$ is the handle metric induced by all handles of codimension greater than two, $g_{\text {can }}$ is the canonical handle metric on $\mathbb{D}^{n}(\pi, \alpha, \omega)$, and $c_{g}$ is a concordance. At the same time, an arbitrary metric $s$ of positive scalar curvature on $\mathbb{T}^{n}(\pi, \alpha, \omega)$ is isotopic to a metric of the form $g_{\text {can }} \cup g_{\text {can }}$. Consider a pairing $\pi_{0}^{c}(n, \pi, \omega) \times$ $\pi_{0}^{c}\left(\mathbb{P S C}\left(M^{n}\right)\right) \rightarrow \pi_{0}^{c}\left(\mathbb{P S C}\left(M^{n}\right)\right)$ which assigns to $[s] \in \pi_{0}^{c}(n, \pi, \omega)$ and $[g] \in \pi_{0}^{c}\left(\mathbb{P S C}\left(M^{n}\right)\right)$ the class $\left[g_{H} \cup c_{g} \cup c_{s} \cup g_{\text {can }}\right]$. It is well defined and constitutes an action by Theorem 1.3 and the Cancellation Lemma.

Let us see that the action is free, i.e., if $[s] \cdot[g]=[g]$, then $s$ is concordant to the standard metric $g_{\text {can }} \cup g_{\text {can }}$ on $\mathbb{T}^{n}(\pi, \alpha, \omega)$. By Theorem 1.1, $[s] \cdot[g]=[g]$ if and only if $g_{H} \cup c_{g} \cup c_{s} \cup g_{\text {can }}$ is concordant to $g_{H} \cup c_{g} \cup g_{\text {can }}$. Theorem 1.3 implies that the concordance is isotopic to a metric of the form

$$
\left(g_{H}+d t^{2}\right) \cup \hat{c} \cup\left(g_{\text {can }}+d t^{2}\right)
$$

where $\hat{c}$ is a relative concordance between $c_{g}$ and $c_{g} \cup c_{s}$. The metric $s$ is concordant to the standard metric on $\mathbb{T}^{n}(\pi, \alpha, \omega)$ if and only if $c_{S}$ is relatively concordant to the trivial concordance $\left.g_{\text {can }}\right|_{\partial D_{n}(\pi, \alpha, \omega)}$. By the Cancellation Lemma,

$$
\left.g_{\text {can }}\right|_{\partial D_{n}(\pi, \alpha, \omega)}+d t^{2} \cong-c_{g} \cup c_{g} \cong-c_{g} \cup c_{g} \cup c_{s} \cong c_{s} \text {. }
$$

Let us now see that the action is transitive. It will be shown that for two arbitrary metrics $g_{0}$ and $g_{1}$ of positive scalar curvature on a $(\pi, \omega)$-manifold $M^{n}$ there is a metric $s$ of positive scalar curvature on $\mathbb{T}^{n}(\pi, \alpha, \omega)$ such that the metric obtained by the action of $s$ on $g_{0}$ is concordant to $g_{1}$. Take representatives $g_{H} \cup c_{g_{0}} \cup g_{\text {can }}, g_{H} \cup$ $c_{g_{1}} \cup g_{\text {can }}$, and $g_{H} \cup c_{s} \cup g_{\text {can }}$ of concordance classes of $g_{0}, g_{1}$, and $s$ respectively. By the Cancellation Lemma, $c_{g_{0}} \cup\left(-c_{g_{0}}\right)$ is relatively concordant to the trivial concordance. Hence, the metric given by the action of $g_{\text {can }} \cup\left(-c_{g_{0}} \cup c_{g_{1}}\right) \cup g_{\text {can }}$ on $g_{0}$ is concordant to $g_{1}$. This completes the proof of Theorem 2.1.

3. Concordance groups of $\mathbb{P S C}$ metrics. This section contains a definition of the concordance groups of metrics of positive scalar curvature, some of their elementary properties, and a proof of the following result.

THEOREM 3.1. If $M^{n}$ is a closed spin manifold of dimension $\geq 6$, then the $i^{\text {th }}$ concordance group $\pi_{i}^{c}\left(\mathbb{P S C}\left(M^{n}\right), g\right)$ of metrics of positive 
scalar curvature on $M^{n}$ is nontrivial for $n+i=4 k+3,8 k, 8 k+1$ where $k \geq 1$ and $i \neq 1,2$.

Let $\{p\}$ stand for the south pole on $S^{i}$, let $d x^{2}$ denote the Euclidean flat metric of $\mathbb{R}^{i}$, and let $g$ be a fixed metric of positive scalar curvature on $M^{n}$.

Construction of the concordance groups of metrics of positive scalar curvature. Let $\pi_{i}^{c}\left(\mathbb{P S C}\left(M^{n}\right), g\right)$ be the set of concordance classes of metrics of positive scalar curvature on $S^{i} \times M^{n}$ which are of the form $d x^{2}+g$ in a neighborhood of $\{p\} \times M^{n}$ in $S^{i} \times M^{n}$. The group operation on $\pi_{i}^{c}\left(\mathbb{P S C}\left(M^{n}\right), g\right)$ is defined as follows. Let $g_{0}$ and $g_{1}$ be representatives of classes $\alpha_{0}, \alpha_{1} \in \pi_{i}^{c}\left(\mathbb{P S C}\left(M^{n}\right), g\right)$. There is a neighborhood $D \times M^{n}$ of $\{p\} \times M^{n}$ in $S^{i} \times M^{n}$ such that $g_{0}, g_{1}$ restricted to it are of the form $d x^{2}+g$. Let $\tilde{g}$ be a metric of positive scalar curvature on $\left(S^{i} \# S^{i}\right) \times M^{n}$ given by identification of the metrics $\left.g_{0}\right|_{\left(S^{i} \times M^{n}\right) \backslash\{p\} \times M^{n}}$ and $\left.g_{1}\right|_{\left(S^{i} \times M^{n}\right) \backslash\{p\} \times M^{n}}$ along the open set $(D \backslash\{p\}) \times M^{n}$. Define $\alpha_{0} \cdot \alpha_{1}$ as the concordance class of the metric $\left(\varphi \times \operatorname{id}_{M}\right)^{*}(\tilde{g})$ where $\varphi$ is a diffeomorphism of $S^{i}$ onto $S^{i} \# S^{i}$ sending the south pole $p$ of $S^{i}$ into a point $q$ of $S^{i}$, \# $S^{i}$ where the metric $\tilde{g}$ is flat. For $i=1$, the set $D \backslash\{p\}$ has two connected components, and it is important from which one the point $q$ is chosen, therefore, for $i=1$, let the point $q$ come from the third quadrant of the first sphere. It is easy to see that the operation is well defined and determines a group structure with the neutral element induced by the class of a metric $g_{\mathrm{fl}}+g$ where $g_{\mathrm{fl}}$ is a metric obtained from the standard sphere metric by making it flat in a neighborhood of the south pole of $S^{i}$.

The groups $\pi_{i}^{c}\left(\mathbb{P S C}\left(M^{n}\right), g\right)$ depend only on concordance class of the diffeomorphism $\varphi$, and this is why they are Abelian for $i>1$. It is easy to see that for concordant metrics $g_{0}, g_{1}$ of positive scalar curvature on $M^{n}$, the groups $\pi_{i}^{c}\left(\mathbb{P S C}\left(M^{n}\right), g_{0}\right)$ and $\pi_{i}^{c}\left(\mathbb{P S C}\left(M^{n}\right), g_{1}\right)$ are isomorphic. One can also check that the map $\pi_{0}\left(\mathbb{P S C}\left(M^{n}\right)\right) \rightarrow$ $\pi_{0}^{c}\left(\mathbb{P S C}\left(M^{n}\right)\right)$ has its analogs $\pi_{i}\left(\mathbb{P S C}\left(M^{n}\right), g\right) \rightarrow \pi_{i}^{c}\left(\mathbb{P S C}\left(M^{n}\right), g\right)$ for $i \geq 1$, which are group homomorphisms.

Proof of Theorem 3.1. Let $M^{n}$ be a simply-connected closed spin manifold and let $i=0$. By Theorem 2.1 there is a bijective correspondence between elements of $\pi_{0}^{c}\left(\mathbb{P S C}\left(M^{n}\right)\right)$ and $\pi_{0}^{c}\left(\mathbb{P S C}\left(S^{n}\right)\right)$. Let $\tilde{\pi}_{0}^{c}\left(\mathbb{P S C}\left(S^{n}\right)\right)$ be the subgroup of $\pi_{0}^{c}\left(\mathbb{P S C}\left(S^{n}\right)\right)$ consisting of concordance classes of those metrics that are boundary restrictions of 
metrics with positive scalar curvature on compact spin manifolds. Hajduk defined a homomorphism $a: \tilde{\pi}_{0}^{c}\left(\mathbb{P S C}\left(S^{n}\right)\right) \rightarrow K O_{n+1}(p t)$ and noticed that $a$ is an isomorphism if the Gromov-Lawson conjecture is true [H1]. Since the conjecture was proved to be true [S] the group $\tilde{\pi}_{0}^{c}\left(\mathbb{P S C}\left(S^{n}\right)\right)$ are nontrivial for $n=4 k+3,8 k, 8 k+1, k \geq 1$. This proves Theorem 3.1 for simply-connected spin manifolds and $i=0$.

If $M^{n}$ be a spin manifold, then the product $S^{i} \times M^{n}$ is spin as well. Therefore it is possible to kill the first two homotopy groups of $S^{i} \times M^{n}$ obtaining a simply-connected spin manifold $W^{n+1}$. When $n+i \geq 6$ the surgery is performed in codimension $\geq 3$ and thus it induces a map $S: \mathbb{P S C}\left(S^{i} \times M^{n}\right) \rightarrow \mathbb{P S C}\left(W^{n+1}\right)$. By Lemma 2.3, if $g_{0}, g_{1} \in \mathbb{P S C}\left(S^{i} \times M^{n}\right)$ are concordant, then $S\left(g_{0}\right), S\left(g_{1}\right)$ are concordant as well, in particular, $S$ induces a map $\pi_{i}^{c}\left(\mathbb{P S C}\left(M^{n}\right)\right) \rightarrow$ $\pi_{0}^{c}\left(\mathbb{P S C}\left(W^{n+1}\right)\right)$. By Theorem 2.1 this map induces another one $\pi_{0}^{c}(n, \pi, 0) \rightarrow \pi_{0}^{c}\left(\mathbb{P S C}\left(S^{n}\right)\right)$, which will be still denoted by $S$. Fix a metric $g$ of positive scalar curvature on $\mathbb{T}^{n}(\pi, \alpha, 0)$. If the connected sum $-S(g) \# g$ is taken outside the support of the surgeries defining $S$, then $S(-S(g) \# g)=-S(g) \# S(g)$. By the Cancellation Lemma, $-S(g) \# S(g)$ is concordant to the standard metric on $S^{n}$. Therefore, for every metric $s$ of positive scalar curvature on $S^{n}$ the image of $s \# S(g) \# g$ under $S$ is concordant to $s$. This completes the proof of Theorem 3.1.

\section{REFERENCES}

[G] P. Gajer, Riemannian metrics of positive scalar curvature on compact manifolds with boundary, Ann. Global Anal. Geom., 5 (1987), 179-191.

[GL1] M. Gromov and H. B. Lawson, Jr., The classification of simply-connected manifolds with positive scalar curvature, Ann. of Math., 111 (1980), 423-432.

[GL2] Positive scalar curvature and the Dirac operator on complete Reimannian manifolds, Publ. IHES, 58 (1983), 295-408.

[H1] B. Hajduk, Metrics of positive scalar curvature on spheres and Gromov-Lawson conjecture, Math. Ann., 280 (1988), 409-415.

[H2] - On the obstruction group to the existence of Riemannian metrics of positive scalar curvature, preprint.

[Hi] N. Hitchin, Harmonic spinors, Adv. in Math., 14 (1974), 1-55.

[SY] R. Shoen and S.T. Yau, On the structure of manifolds of positive scalar curvature, Manuscripta Math., 28 (1979), 159-183.

[S] S. Stalz, Simply connected manifolds with positive scalar curvature, preprint.

Received April 4, 1991 and in revised form February 13, 1992. 


\title{
PACIFIC JOURNAL OF MATHEMATICS
}

\author{
Founded by
}

E. F. BECKENBACH (1906-1982) F. WoLF (1904-1989)

\section{EDITORS}

$\begin{array}{ll}\begin{array}{l}\text { V. S. VARADARAJAN } \\ \text { (Managing Editor) }\end{array} & \begin{array}{l}\text { NiCHOLAS ERCOLANI } \\ \text { University of Arizona }\end{array} \\ \text { University of California } & \text { Tucson, AZ 85721 } \\ \text { Los Angeles, CA 90024-1555 } & \text { ercolani@math.arizona.edu } \\ \text { vsv@math.ucla.edu } & \text { R. FinN } \\ \text { HERBERT CLEMENS } & \text { Stanford University } \\ \text { University of Utah } & \text { Stanford, CA 94305 } \\ \text { Salt Lake City, UT 84112 } & \text { finn@gauss.stanford.edu } \\ \text { clemens@math.utah.edu } & \text { VAUGHAN F. R. JoNEs } \\ \text { F. MichAEL CHRIsT } & \text { University of California } \\ \text { University of California } & \text { Berkeley, CA 94720 } \\ \text { Los Angeles, CA 90024-1555 } & \text { vfr@math.berkeley.edu } \\ \text { christ@math.ucla.edu } & \text { STEVEN KERCKHoFF } \\ \text { THomas ENRIGHT } & \text { Stanford University } \\ \text { University of California, San Diego } & \text { Stanford, CA 94305 } \\ \text { La Jolla, CA 92093 } & \text { spk@gauss.stanford.edu } \\ \text { tenright@ucsd.edu } & \end{array}$

\author{
C. C. MOORE \\ University of California \\ Berkeley, CA 94720
}

MaRTin ScharlemanN

University of California

Santa Barbara, CA 93106

mgscharl@henri.ucsb.edu

\author{
HAROLD STARK \\ University of California, San Diego \\ La Jolla, CA 92093
}

\begin{tabular}{ll}
\multicolumn{1}{c}{ SUPPORTING } & INSTITUTIONS \\
UNIVERSITY OF ARIZONA & UNIVERSITY OF OREGON \\
UNIVERSITY OF BRITISH COLUMBIA & UNIVERSITY OF SOUTHERN CALIFORNIA \\
CALIFORNIA INSTITUTE OF TECHNOLOGY & STANFORD UNIVERSITY \\
UNIVERSITY OF CALIFORNIA & UNIVERSITY OF HAWAII \\
MONTANA STATE UNIVERSITY & UNIVERSITY OF TOKYO \\
UNIVERSITY OF NEVADA, RENO & UNIVERSITY OF UTAH \\
NEW MEXICO STATE UNIVERSITY & WASHINGTON STATE UNIVERSITY \\
OREGON STATE UNIVERSITY & UNIVERSITY OF WASHINGTON
\end{tabular}

The Supporting Institutions listed above contribute to the cost of publication of this Journal, but they are not owners or publishers and have no responsibility for its content or policies.

Mathematical papers intended for publication in the Pacific Journal of Mathematics should be in typed form or offset-reproduced (not dittoed), double spaced with large margins. Please do not use built up fractions in the text of the manuscript. However, you may use them in the displayed equations. Underline Greek letters in red, German in green, and script in blue. The first paragraph must be capable of being used separately as a synopsis of the entire paper. In particular it should contain no bibliographic references. Please propose a heading for the odd numbered pages of less than 35 characters. Manuscripts, in triplicate, may be sent to any one of the editors. Please classify according to the 1991 Mathematics Subject Classification scheme which can be found in the December index volumes of Mathematical Reviews. Supply name and address of author to whom proofs should be sent. All other communications should be addressed to the managing editor, or Julie Speckart, University of California, Los Angeles, California 90024-1555.

There are page-charges associated with articles appearing in the Pacific Journal of Mathematics. These charges are expected to be paid by the author's University, Government Agency or Company. If the author or authors do not have access to such Institutional support these charges are waived. Single authors will receive 50 free reprints; joint authors will receive a total of 100 free reprints. Additional copies may be obtained at cost in multiples of 50 .

The Pacific Journal of Mathematics (ISSN 0030-8730) is published monthly except for July and August. Regular subscription rate: $\$ 190.00$ a year (10 issues). Special rate: $\$ 95.00$ a year to individual members of supporting institutions.

Subscriptions, orders for numbers issued in the last three calendar years, and changes of address should be sent to Pacific Journal of Mathematics, P.O. Box 969, Carmel Valley, CA 93924, U.S.A. Old back numbers obtainable from Kraus Periodicals Co., Route 100, Millwood, NY 10546.

The Pacific Journal of Mathematics at P.O. Box 969, Carmel Valley, CA 93924 (ISSN 0030-8730) is published monthly except for July and August. Second-class postage paid at Carmel Valley, California 93924, and additional mailing offices. Postmaster: send address changes to Pacific Journal of Mathematics, P.O. Box 969, Carmel Valley, CA 93924.

PUBLISHED BY PACIFIC JOURNAL OF MATHEMATICS, A NON-PROFIT CORPORATION Copyright $(\mathcal{1} 1993$ by Pacific Journal of Mathematics 


\section{PACIFIC JOURNAL OF MATHEMATICS}

Volume $157 \quad$ No. $2 \quad$ February 1993

Strong integral summability and the Stone-Čech compactification of the half-line

JEFF CONNOR and MARY ANNE SWARDSON

The endlich Baer splitting property

225

THEODORE GERARD FATICONI

The formal group of the Jacobian of an algebraic curve

MARGARET N. FREIJE

Concordances of metrics of positive scalar curvature

PAWEL GAJER

Explicit construction of certain split extensions of number fields and constructing cyclic classfields

STANLEY JOSEPH GURAK

Asymptotically free families of random unitaries in symmetric groups

ALEXANDRU MiHAi NiCA

On purifiable subgroups and the intersection problem

TAKASHI OKUYAMA

On the incidence cycles of a curve: some geometric interpretations

LUCIANA RAMELLA

On some explicit formulas in the theory of Weil representation

R. RANGA RAO

An analytic family of uniformly bounded representations of a free product of 373 discrete groups

JANUSZ WYSOCZAŃSKI

Errata: "Dentability, trees, and Dunford-Pettis operators on $L_{1}$ "

MARIA GIRARDI and ZHIBAO HU

Errata: "Poincaré cobordism exact sequences and characterisation" 